\title{
Comunicación

\section{Historia y comunicación social: apuntes para un diálogo inconcluso. Aproximación crítica al campo de estudios históricos en comunicación}

JANNY AMAYA TRUJILLO1

El propósito fundamental de este ensayo es ofrecer un balance crítico en torno al estatuto disciplinar de los estudios históricos en comunicación y su posición dentro del campo investigativo de la comunicación social. Se trata de observar, además, sus deudas constitutivas con respecto a la historiografía y evidenciar algunas de las posibles constricciones y condicionamientos heredados y reproducidos en este ámbito de estudios. Por último, se sintetizan algunos de los desafíos estratégicos que enfrenta esta área de estudios, así como sus implicaciones epistemológicas para el campo científico de la comunicación.

PALABRAS CLAVE: historia, comunicación social, estudios históricos en comunicación.
The central purpose of this article is to offer a critical approach to the development of the historical studies in communication, and its position into the communication research field. Also, the author tries to show some of the most important limitations and constrictions inherited and reproduced in this field of studies. It offers a synthesis of the main challenges of this area, and its epistemological projections to the communication field.

KEY WORDS: history, communication, historical studies in communication.

1 Universidad de la Habana, Cuba.

Correo electrónico: jannyamaya@gmail.com 
No son pocos los debates actuales que giran en torno al estatuto disciplinar de los estudios sobre comunicación. Campo científico relativamente joven y empeñado en dilucidar un fenómeno tan antiguo como el hombre mismo, que se constituye -en virtud de la complejidad de su propio objeto- en una zona de estudios marcada por la convergencia, el entrelazamiento y la hibridación de diversos enfoques y perspectivas disciplinares.

El ámbito específico de los estudios históricos en comunicación es, quizás, uno de los dominios donde el desdibujamiento de estas fronteras se hace más evidente. Signado por la que algunos denominan paradójica ambigüedad entre la antigüedad de las investigaciones fundacionales de esta área y su reciente -e inconclusa- articulación disciplinar, los estudios históricos en comunicación no escapan a la fragmentación y dispersión que caracterizan al campo de la comunicación en general. El propósito fundamental de este ensayo es ofrecer un balance crítico sobre el estatuto disciplinar de los estudios históricos en comunicación y su posición dentro del campo investigativo de la comunicación social.

En tanto una de las intenciones fundamentales de este trabajo consiste en mostrar las polémicas alrededor del estatuto disciplinar de este ámbito de estudios, optamos por asumir la denominación genérica de campo de estudios históricos en comunicación, en lugar de otra que pueda significar su designación apriorística como disciplina científica consolidada. En este sentido, nos afiliamos a las consideraciones de los investigadores mexicanos Raúl Fuentes Navarro y Enrique Sánchez Ruiz (Fuentes \& Navarro, 1992), quienes - retomando los planteamientos del norteamericano Wilbur Schramm- sostienen la idea de que más que una disciplina científica, la investigación en comunicación puede ser considerada como un campo "en un sentido más sociológico que epistemológico: tenemos objetos de estudio (...) y una comunidad científica que se interesa sistemáticamente por los mismos" (Sánchez, 2002, p. 26).

Tomando como punto de partida estos referentes, nuestra propuesta de asumir la denominación de campo de estudios históricos en comunicación se dirige prioritariamente hacia la identificación genérica de una tradición investigativa que ha centrado su atención fundamental en el análisis, desde una perspectiva histórica, de las prácticas, instituciones y sistemas de comunicación. Se refiere, en suma, a la práctica 
científica de la investigación histórica en el campo de la comunicación. Esta precisión resulta necesaria en tanto enfoca nuestro análisis en un subcampo especifico dentro de lo que ha sido definido como campo científico de la comunicación.

Las técnicas de investigación desplegadas fueron tres: la investigación bibliográfica, el análisis de contenido cualitativo del discurso científico sobre el campo de estudios históricos en comunicación ${ }^{2}$ y la entrevista a expertos. A partir de ellas se intenta ofrecer no un diagnóstico exhaustivo sino un cuadro indicativo de los rasgos y tendencias más acusados de este campo de estudios.

\section{CONSIDERACIONES PRELIMINARES}

PARA ADENTRARSE EN UN DOMINIO HÍBRIDO

El propósito de emprender el análisis de los genéricamente denominados estudios históricos en comunicación compromete, de entrada, una serie de reconocimientos imprescindibles, vinculados, en primer término, a la propia ambigüedad del estatuto disciplinar de esta área del conocimiento y la investigación. En virtud de su ubicación intersticial, el análisis de los denominados estudios históricos en comunicación debe ser esbozado sólo a partir del reconocimiento de las condiciones epistemológicas básicas de ambos campos disciplinares: la historiografía y la(s) ciencia(s) de la comunicación. Dichas condiciones constituyen sobredeterminantes esenciales en la articulación de este ámbito de estudios.

De una parte, la cuestión de la identidad o autonomía disciplinar de la comunicación, en cuanto campo, así como sus coincidencias con otras disciplinas sociales ha sido considerada una polémica vigente:

2 Es decir, el conjunto de balances críticos y aproximaciones descriptivas al origen, desarrollo y estado actual de este campo de estudios, contenido en artículos, ponencias y libros. Estos balances han sido fruto, en su mayoría, de ejercicios de autoobservación por parte de los propios investigadores de esta área de estudios en Iberoamérica y, en menor medida, en Estados Unidos, quienes proporcionan, en su conjunto, un panorama indicativo - y valorativo- de las tendencias fundamentales observadas dentro del campo. 
Hay quien propone que no se considere a la comunicación una ciencia o disciplina, puesto que no tiene principios explicativos propios, $[\mathrm{y}]$ sigue modelos teóricos importados de otras disciplinas. Hay también quienes aceptan la existencia de una disciplina llamada comunicación, si bien señalan su falta de autonomía como campo de conocimiento (Capparelli \& Stumpf, 2001, p. 64).

En el proceso de constitución del campo de estudios de la comunicación se ha observado la convergencia simultánea de movimientos centrífugos -que resultan del interés de otros campos de conocimiento por el estudio de procesos y fenómenos comunicativos-, y movimientos centrípetos, que expresan la apropiación por parte de especialistas del campo de la comunicación de perspectivas analíticas de otras áreas del saber (ibidem, p. 67).

No obstante, puede afirmarse que la comunicación se encuentra aún en medio de un debate sobre su estatuto dentro de las ciencias sociales:

La cercanía de la comunicación con las demás disciplinas sociales ha constituido quizás el mayor impedimento para lograr esa ansiada independencia, en un campo científico que posiblemente tampoco pueda fundamentar esas divisiones disciplinarias, muchas de ellas heredadas del siglo pasado (Gil, 2001, p. 92).

Por otro lado, pese a la antigüedad de la historiografía como campo de conocimiento, algunos autores reconocen que su fundamentación teórico-epistemológica parece encontrarse, aún hoy, en un estado de menor articulación con respecto a otras ciencias sociales tradicionales. Según explicita Aróstegui (2001, p. 29-32), este cierto retraso teórico metodológico puede obedecer a la concomitancia de varios factores complejos, como la naturaleza de su propio objeto de estudio, la función social e ideológica de este tipo de conocimiento, tradicionalmente asociado o subordinado al ejercicio del poder y, por último, a la propia actitud de resistencia ante la reflexión teórica largo tiempo predominante entre los propios historiadores.

Sin embargo, en el ambiguo estatuto disciplinario de los estudios históricos en comunicación han incidido, además, otros aspectos rela- 
cionados con el escaso reconocimiento de este ámbito de estudios por parte de la historiografía. De modo decisivo, ha repercutido también la exigüidad de los esfuerzos en la propia delimitación teórico-epistemológica de este ámbito de estudios.

Resulta innegable la existencia de una larga tradición de investigación que ofrece hoy un conjunto numeroso de investigaciones históricas de distinto rango y enfoques, aplicadas al estudio de prácticas, instituciones y medios de comunicación. No obstante, es preciso reconocer que ella no ha tenido como correlato el desarrollo de un corpus teórico, ni -hasta décadas recientes- la voluntad manifiesta de su articulación como disciplina en el campo de la comunicación (Román, 2000; Montero \& Rueda, 2001).

Por otra parte, ha sido reconocido que, pese a la doble procedencia disciplinar de este campo de estudios, su estatuto actual parece ser el resultado de un proceso en el que ambas disciplinas, la historiografía y la(s) ciencia(s) de la comunicación " han avanzado de espaldas la una a la otra" (Yanes, 2003, p. 242).

De modo general, resulta perceptible, dentro del campo, un cierto estatuto de marginalidad con respecto a las disciplinas que le dieron origen. Es decir, un insuficiente reconocimiento desde el ámbito de la historiografía y -aunque en menor medida- también observable dentro del propio campo de la comunicación.

Es esta una noción predominante, una especie de núcleo consensual en la percepción que comparten sobre el campo de los estudios históricos en comunicación algunos de sus más diversos exponentes. Para John Nerone, del Instituto de Investigación de la Comunicación de la Universidad de Illinois, en Estados Unidos, es evidente que "la comunidad de historiadores no ha reconocido que esta obra tenga su propia identidad o su propia temática"3 (Nerone, s/a).

Un argumento parecido maneja la mexicana Celia del Palacio Motiel (2000). Según esta autora, si bien ha sido considerada una condición de triple marginalidad de los estudios sobre comunicación en América Latina ("marginalidad, en primer lugar, de la investigación científica, margi-

3 Traducción del inglés de Hilda Bello Quintana. Biblioteca Central Rubén Martínez Villena, Universidad de La Habana (2007). 
nalidad de las ciencias sociales y humanidades frente al poder y prestigio de las ciencias 'duras', marginalidad del campo de investigaciones en comunicación entre las ciencias sociales" (Sánchez, citado en Del Palacio, 2000), es posible reconocer, para los historiadores de los medios aún otro nivel de marginalidad. "Me atrevo a afirmar que los historiadores de los medios están todavía más alejados de ese espacio marginal que Sánchez Ruiz imagina para los comunicólogos", afirma esta autora.

En alguna medida, el insuficiente reconocimiento a este campo de investigación, por parte de la historiografía, puede ser atribuido al rezago de concepciones positivistas, así como a ciertas nociones subyacentes en la definición misma del propio término de comunicación. Como argumentara Michael Schudson (1993):

En parte, la razón se encuentra en el hecho de que los medios de comunicación son considerados en una amplia medida, [...] los transmisores, mas no los creadores de las causas y los efectos de los que, por lo general, se ocupan los historiadores. [...] Los historiadores profesionales, a causa de su entrenamiento, son resistentes a las epistemologías del método histórico o a las prácticas de investigación histórica que situarían el fondo en primer plano.

Es posible constatar, también, un cierto soslayo o desconocimiento de los estudios históricos en el propio campo de la comunicación.

Todos los que nos dedicamos a la historia de la comunicación en su sentido más amplio, sabemos que en el campo de las ciencias de la comunicación, predomina el punto de vista sincrónico. Sin embargo, estamos convencidos de que el componente diacrónico es un elemento esencial para comprender el fenómeno de la comunicación en todos sus aspectos (Pizarroso, 1998, p. 161).

Para algunos autores, los motivos de este desinterés pueden vincularse al predominio tradicional de marcos sociológicos en la investigación en comunicación.

Según explicita la investigadora brasileña Barbosa (2005): 
En el campo de la comunicación predomina una cierta ojeriza, o un cierto miedo de la historia [...]. La sociología es el bies dominante en términos conceptuales de los estudios de comunicación. Entonces, lo que acontece es una deshistorización de los temas de comunicación. [...] Creo que ese es el gran pecado de los estudios de comunicación: no historizar sus procesos (p. 142). ${ }^{4}$

Según se infiere de estas consideraciones, se podrían estar reproduciendo, artificialmente dentro del propio campo de la comunicación, las escisiones y contraposiciones tradicionales entre ambas disciplinas científicas -inscritas desde su propia constitución como ámbitos de conocimiento-, toda vez que han proporcionado marcos disciplinares básicos en la constitución del campo científico de la comunicación.

Más allá de las distintas consideraciones en torno al estatuto de legitimidad conferido a los estudios históricos en comunicación desde ambos campos matriciales -la historiografía y las ciencias de la comunicación-, los debates sobre la definición de su propio objeto de estudios pueden resultar indicativos del estatuto actual, así como de la naturaleza misma de este proyecto disciplinar. Una doble topografía ha signado la articulación de este campo de estudios. De una parte, esta ha estado determinada en función de las distintas concepciones alrededor de su objeto. Por otro lado, su proceso de configuración ha estado también demarcado por la influencia de los diversos paradigmas historiográficos. Siguiendo esta doble lógica - no disociada, sino superpuesta- se estructuran, entonces, los siguientes epígrafes.

\section{ESTUDIOS HISTÓRICOS EN COMUNICACIÓN O HISTORIA}

DE LA COMUNICACIÓN SOCIAL: ALGUNOS DEBATES

Y POSTURAS SOBRE LA IDENTIDAD

DE UN (SUB) CAMPO DISCIPLINAR

"La historia de la comunicación es una disciplina joven, en la que se está trabajando desde hace relativamente poco tiempo. De he-

4 Todas las citas de Marialva Barbosa que aparecen en este artículo han sido traducidas del portugués por ella misma. 
cho, ni siquiera existe un acuerdo para designarla (...) o para definir su contenido y alcance" asevera la investigadora española Moreno (1997, p. 13) lo que podría ser una síntesis lapidaria del estatuto actual de los estudios históricos en comunicación. Las consideraciones en torno a la delimitación de su objeto de estudio han involucrado, en sí mismas, algunas discusiones que atañen directamente a la pertinencia de su definición como (sub)campo o disciplina específica, en contraste con las tendencias a disgregar o diluir el análisis de las prácticas instituciones o sistemas de comunicación en el marco de la historia general.

Es posible identificar dos posturas básicas sobre las cuales han fluctuado las consideraciones sobre el objeto de estudio de la historia de la comunicación: una generalista, que considera su inclusión en el marco de la historia, como mera derivación o rama de una historia general, y otra que demanda su especificidad como materia autónoma, dotada de singularidades temáticas y cronológicas, y que postula su identidad como proyecto disciplinar con respecto a otras vertientes tradicionales de la historia (Gómez \& Tresserras citados en Del Palacio, 2000; Montero, et al., 2001; Román, 2000; Yanes, 2003).

Desde la primera de estas posturas se plantea, genéricamente, que una historia general es suficiente para dar cuenta de la evolución o el devenir de los procesos, prácticas, instituciones y sistemas comunicativos. Es decir, se defiende la hipótesis de que no resulta necesario prestar una atención específica a las transformaciones en el ámbito de la comunicación, toda vez que dichas transformaciones son sólo el resultado de $-y$ por lo tanto resultan suficientemente explicadas por- el cambio histórico en general. En este sentido, la comunicación social queda reducida al rango de un epifenómeno.

De otra parte, se sitúan, entonces, aquellos que defienden la existencia de la historia de la comunicación como un dominio autónomo, cuyo objeto de estudio no resulta agotado desde otras vertientes historiográficas. Desde esta posición, se ha abogado por la consideración de la historia de la comunicación social como subcampo o disciplina específica dentro del campo de la comunicación, aun cuando se reconoce la necesaria existencia de una relación interdisciplinar con el campo de la historiografía (Barbosa, 2005; Gómez \& Tresserras citados en Del 
Palacio, 2000; Montero, et al., 2001; Nerone, s/a; Pizarroso, 1998; Román, 2000; Schudson, 1993).

Sin embargo, como se ha señalado, la voluntad de su articulación como disciplina autónoma se ha expresado más en el desarrollo -diverso y, en ocasiones, disperso- de investigaciones históricas puntuales que en su necesaria fundamentación teórico-epistemológica. En tal sentido se ha puntualizado que:

El conjunto de las aportaciones individuales que hasta ahora han confluido en el estudio de la historia de la comunicación social no son capaces de encerrar o delimitar por sí solas el objeto de estudio de esa disciplina (Montero, et al., 2001, p. 48).

Ello se ha traducido en ciertos rasgos de entropía en la propia definición de este ámbito de estudios. En muchos casos, la denominación de "historia de la comunicación", ausente de definiciones explícitas, parece remitir a una suerte de consenso tácito, que se refiere a "la actividad periodística pasada", el "desarrollo cultural pasado", o simplemente, en su sentido más amplio, a "cualquier actividad humana pasada relativa a la dimensión comunicativa" (Atwood, 1978, p. 8 , traducción de la autora). Aunque en este sentido la orientación temporal/ diacrónica es básica, resulta insuficiente para el establecimiento de criterios de delimitación disciplinar, ya que ello debe partir de una adecuada definición de categorías centrales y distintivas como requisito indispensable para su consolidación como disciplina, así como para la comprensión de su propio lugar en la actividad científica y académica en el campo de la comunicación.

Esta entropía se ha expresado, también, en la diversidad de posturas -explícitas o implícitas- desde las cuales se ha proyectado la comprensión y el establecimiento de su campo y objeto de estudio. Al respecto, la investigadora española María José Ruiz Acosta observa cómo este campo se ha convertido en:

Un espacio donde convergen las más agrias disputas, las tesis más encontradas o las más claras oposiciones entre los investigadores; discrepancias en cuyo seno se observan - es evidente- los más diversos pareceres acerca de la naturaleza misma del fenómeno comunicativo (1998, p. 398). 
Según esta autora es posible reconocer en este campo la existencia de tres perspectivas predominantes en la definición de su objeto de estudio. La primera de ellas parte de una sobrevaloración de la racionalidad tecnológica como instancia decisiva y explicativa del devenir históricosocial, y centra su atención en la evolución de los sistemas tecnológicos que garantizan la producción y circulación de mensajes. La segunda asume como eje central en la delimitación de su objeto de estudio la aparición de instituciones y figuras profesionales especializadas exclusivamente en actividades informativas y comunicativas, restringiendo la noción de comunicación social a la emergencia de instituciones y prácticas esencialmente modernas. La tercera propone, en cambio, la comprensión de las prácticas, procesos e instituciones comunicativas desde una perspectiva sociocultural, restituyendo la complejidad de sus interrelaciones con el devenir histórico-social.

Incluso en algunos autores que se adscriben abiertamente a la última de las tendencias mencionadas (Atwood, 1978; Montero, et al., 2001; Ruiz, 1998), se evidencia uno de los rasgos más acusados y problemáticos en la definición de este ámbito de estudios: la propensión a adoptar objetos preconstruidos por el lenguaje común. La noción de medios de comunicación -asumida con frecuencia como sobreentendida, no construida o suficientemente conceptualizada- continúa siendo un eje central en algunos trabajos que se proponen la delimitación teórica del campo de los estudios históricos en comunicación. Precisamente, esta tendencia ha sido considerada "un obstáculo epistemológico ampliamente notado en la investigación en comunicación" (Vassallo, 1999, p. 20). Ello ha conducido a una suerte de "efecto de obviedad", que tiende a eludir operaciones de ruptura epistemológica capaces de establecer distinciones fundamentales entre objetos reales y objetos de la ciencia, concebidos como sistemas de relaciones expresamente construidos.

De las consideraciones esbozadas acerca de la polémica definición de este (sub)campo de estudios emergen, entonces, una serie de observaciones básicas que muestran algunas constricciones fundamentales perceptibles en la comprensión de la historia de la comunicación como proyecto disciplinar. Si, como plantea genéricamente esta última perspectiva, se trata de rescatar y reconocer la dimensión sociocultural y 
la historicidad misma de los procesos y fenómenos comunicacionales, entonces ello debe partir de la mencionada necesidad de:

Sustituir el concepto predominante que identifica a la comunicación con la transmisión y circulación social de mensajes por un marco conceptual más complejo, alrededor de la comunicación considerada como proceso sociocultural básico, es decir, como producción de sentido (Fuentes, 1999, p. 58-59).

Sólo a partir de la reformulación consciente de algunos principios de inteligibilidad fuertemente entronizados en la concepción misma de su objeto podrá considerarse no sólo la madurez de este ámbito de estudios, sino también sus contribuciones básicas al campo científico de la comunicación.

Proponemos, entonces, una mirada retrospectiva hacia su génesis, así como a las principales tendencias de esta tradición investigativa, ejercicio que puede arrojar algunas luces esclarecedoras acerca de las señas de identidad de este (sub)campo disciplinar.

\section{PARA COMPRENDER LOS MOTIVOS DE UN DEBATE: UNA MIRADA A LA TRADICIÓN INVESTIGATIVA}

La antigüedad de las investigaciones fundacionales en el campo de los estudios históricos en comunicación remite al contexto de emergencia de la prensa como fenómeno masivo a partir de las últimas décadas del siglo XIX. Bajo el signo de la letra impresa se abriría una serie de interrogantes en torno a la prensa como fenómeno social de proporciones crecientes.

Se ha convenido en que la aparición de las primeras investigaciones de este tipo pueden situarse como los antecedentes inaugurales del propio campo de estudios de la comunicación, precediendo en varias décadas las primeras reflexiones teóricas sobre el fenómeno de la comunicación de masas. No obstante, el reconocimiento de esta vertiente de estudios como una de las matrices fundacionales del campo de la comunicación, aparece frecuentemente eclipsado en contraste con la centralidad que luego alcanzarían las aportaciones de otras ciencias sociales, como la psicología y la 
sociología en el estudio de la comunicación. Así, suele en ocasiones desconocerse su preexistencia, o bien asumirla como un estadio precientífico de la investigación en comunicación.

Es necesario admitir que las orientaciones conceptuales y metodológicas de estos estudios estuvieron lógicamente vinculadas a las tendencias positivistas, predominantes en la disciplina historiográfica de la época. Al respecto, Román puntualiza que:

El nacimiento de la historia de la comunicación está vinculado al desarrollo de la propia historia, sobre todo la contemporánea. En la medida en que los diversos aspectos de la comunicación tuvieron un protagonismo cada vez más claro, los historiadores de la política, de la literatura o de las ideas iniciaron las investigaciones en este campo (2000, p. 125-126).

Se ha distinguido como un sesgo predominante entre estos primeros estudios sobre la historia de la prensa, su función políticamente orientada hacia el reforzamiento de las ideologías nacionales:

[...] la necesidad de autoafirmación de los Estados empujó, entre otros aspectos, al desarrollo de "historias oficiales del periodismo nacional", utilizando las fronteras como límites científicos y los papeles y periódicos como material justificativo de ese nacionalismo por lenguaje, difusión e idiosincracia (Timoteo, 1988, p. 11).

Puede considerarse que el proceso de emergencia e institucionalización de los estudios históricos en el campo de la comunicación estuvo encauzado -en términos generales- según una doble funcionalidad. De una parte, funcionalidad política orientada a la consolidación y legitimación de ideologías nacionales, y de otra, funcionalidad en pro de la afirmación y “dignificación” de la propia actividad periodística, a partir del registro de sus antecedentes fundacionales y de la presentación de modelos ejemplares en el ejercicio de la profesión.

Más allá de ciertas diferencias en cuanto a su organización institucional, quedarían esbozadas algunas tendencias que orientarían el campo de los estudios históricos en comunicación y que resultarían predominantes durante buena parte del siglo XX: "Se comenzó a estructurar una discipli- 
na cuyo peso fundamental estuvo centrado en la historiografía" (Román, 2000, p. 126).

El marcado influjo de la historiografía positivista, en la emergencia de este campo de estudios, orientaría algunas tendencias básicas, todavía perceptibles. Entre ellas se reconoce, por ejemplo, su propensión a constreñir el análisis histórico de las prácticas y procesos comunicativos desde un enfoque que ha privilegiado casi exclusivamente las prácticas mediáticas de comunicación, centrándose prioritariamente en el devenir de las instituciones de comunicación masiva o en el desarrollo de los soportes tecnológicos que posibilitaron su articulación. Esta última ha sido una tendencia marcada que ha conducido, incluso, a comparar el estado de la disciplina con "un museo de tecnología" (Herrera, 2001, p. 14).

No obstante, una de las huellas más perceptibles de la influencia positivista ha sido la reticencia ante un interés sistemático por la reflexión teórica dentro de su propio campo de estudios. La comprensión de las dimensiones teóricas de la historia de la comunicación ha estado frecuentemente cargada de problemas fundamentales asociados al paradigma historiográfico positivista. Ello ha condicionado que "la mayoría de los historiadores de la comunicación ha considerado a la teoría como una entidad separada de la propia historia, más que como parte integral de los procesos históricos" (Brennen, 1993, p. 95). Al respecto, son esclarecedores los reclamos lanzados por el norteamericano Roy Atwood a fines de la década de 1970, dirigidos a la vertiente específica de la historia de la prensa. En este sentido afirmaría que:

La propuesta de nuevas rutas para el desarrollo de los estudios históricos del periodismo no será posible a menos que los historiadores rompan de una vez y por todas con esa problemática escisión entre la teoría y la práctica, y comiencen por la construcción de sólidos cimientos teóricos para sus análisis históricos (Atwood, 1978, p. 9).

Otra de las huellas más significativas del paradigma positivista se ha expresado en la tendencia a la fragmentación de este campo de estudios, a partir de la delimitación de objetos puntuales y áreas específicas, insuficientemente articulados desde perspectivas integradoras u holísticas. 
En consonancia con los aires renovadores en el campo de la historiografía, aunque tardíamente apropiados en el campo de la historia de la comunicación, comenzarían a vislumbrarse algunas alternativas fundamentales al modelo positivista hegemónico. En este sentido, se ha destacado, por ejemplo, las aportaciones que en el orden teórico-epistemológico encerraba el proyecto historiográfico de la Escuela de los Annales para la propia historia de la comunicación. A partir de su propia concepción de la historia como totalidad, en sus principios programáticos abiertos a la interdisciplinariedad, así como en el interés por el abordaje analítico de ámbitos socioculturales más amplios, la propuesta "annalista" pudo constituirse en un emplazamiento básico para la legitimación y consolidación del campo de la historia de la comunicación social.

Desde los postulados de una historia total:

Podía reivindicarse una historia de la comunicación social como manto integrador en el que confluyesen otras variables históricas aparentemente desligadas entre sí. Otro influjo de la historia total se tradujo en insistir en la necesidad de abordar el estudio de los medios de comunicación desde todos los condicionantes en que se inscribían, ya fuesen jurídicos, político-institucionales o económicos (Montero, et al., 2001, p. 74).

Sin embargo, la propia condición de inmadurez de este ámbito de estudios retardaría considerablemente las posibilidades de establecer un diálogo que pudo resultar más fecundo para ambos campos disciplinares. En tanto la apertura de esta escuela historiográfica sería notable hacia otras disciplinas consolidadas, el propio desdibujamiento del campo de estudios de la comunicación influiría en un aplazamiento de sus contribuciones fundamentales.

En cambio, los influjos más inmediatos de la propuesta historiográfica de los Annales para este campo provendrían de la denominada "historia serial", otra de las vertientes más significativas de dicha escuela. Es en esta corriente que puede considerarse el auge de los estudios morfológicos de la prensa, paralelo al desarrollo alcanzado en el trabajo con la serialización estadística en el campo de la historiografía. Esta tendencia asumiría como referente fundamental las propuestas metodológicas for- 
muladas por el francés Jacques Kayser (1961). Interesado en aspectos concernientes a la presentación, constitución y contenidos de la prensa periódica, este autor articularía su propuesta con la aspiración de "medir" y "comparar" los rasgos morfológicos de los periódicos (ibidem, p. 11).

Pero este aparente desplazamiento -que llegaría a proporcionar incluso la ilusión de una autonomía científica en el estudio de la prensa- no implicaba realmente una complejización analítica para el campo de la historia de la comunicación, sino un traslado desde el énfasis previamente otorgado a la exposición cronológica del devenir de los medios, atendiendo a sus rasgos institucionales y tecnológicos, hacia un interés marcado en el estudio de sus mensajes y en el tratamiento otorgado a coyunturas o situaciones históricas significativas, aunque desde una perspectiva igualmente descriptiva y escasamente preocupada por explicar sus interrelaciones con las dinámicas sociohistóricas.

No obstante, y aunque de modo un tanto tardío, una de las apropiaciones fundamentales de los postulados "annalistas" en el campo de la historia de la comunicación sería precisamente el de su reformulación de la noción de la temporalidad en los análisis históricos. En este sentido, resultaría significativa la influencia braudeliana para el establecimiento de criterios de delimitación temporal pertinentes para la historia de la comunicación, desde una perspectiva que complejizaba la cronología articulada sobre grandes hechos y fechas como criterio metodológicamente predominante. A partir de las consideraciones braudelianas, según las cuales el análisis histórico debía partir de un reconocimiento del carácter diferencial de los tiempos históricos operando sobre un criterio temporal triple -tiempo largo o estructural, tiempo medio o coyuntural, y un tercero breve, corto o de evento-, el investigador español Jesús Timoteo Álvarez (1988), validaría la posibilidad de concebir criterios de periodización específica para el estudio de la historia de la comunicación.

Sin embargo, en términos generales, se ha señalado cómo a pesar de que las propuestas "annalistas" supusieron un giro de gran impacto en el desarrollo de la disciplina historiográfica, sus apropiaciones en el campo específico de la historia de la comunicación pueden considerarse bastante discretas. "No conocemos, sin embargo, una revisión severa de las historias de los medios informativos, una historia que, como querría Bloch, observe mentalidad, lenguaje, hiciera comparaciones, estudie las condiciones materiales" (Gargurevich, 2000). 
El marxismo, como una de las corrientes de mayor impacto en el ámbito de las ciencias sociales, dejaría también una impronta significativa en el campo de los estudios históricos en comunicación. Aun cuando resultan escasas las valoraciones críticas sobre la influencia de este paradigma en el campo de los estudios históricos en comunicación, los españoles Montero (et al., 2001, p. 59-60) consideran que el determinismo de lo material y las relaciones sociales sobre el aspecto ideológico y subjetivo representan dos vectores básicos sobre los que se articularán las reflexiones de la historiografía marxista de la comunicación.

La apropiación de este sistema de ideas en el campo específico de la historia de la comunicación estuvo en buena medida asociada a lecturas deterministas, vinculadas con lo que se ha identificado como el "marxismo vulgar". Desde esta perspectiva, se establecería una postura analítica -no exclusiva, aunque predominante-según la cual el lugar de los procesos comunicativos en las dinámicas sociohistóricas quedaría restringido a la producción y reproducción de la ideología dominante. El análisis de los medios de comunicación, y su proyección como instrumentos de legitimación o emancipación social, vincularía estrechamente los estudios sobre la historia de la prensa con otras nociones centrales como las de ideología, lucha de clases, reproducción social, y construcción del consenso (ibidem, p. 60). Se ha reconocido la presunción implícita, en estos estudios, de una correspondencia directa entre intereses dominantes y contenido de los productos comunicativos.

Pueden valorarse aportes generales de la apropiación de la perspectiva marxista a partir de la reinserción de las cuestiones relacionadas con el poder, los conflictos clasistas, la construcción de la hegemonía y la atención a grupos sociales hasta entonces marginados de los estudios históricos en el campo de la comunicación.

De una narrativa enciclopédica no comprometida, acrítica, se pasó al rescate de visiones críticas ignoradas, a la búsqueda de nuevas fuentes, datos, que inauguraron una narrativa distinta de la historia de la información y de los medios (Gargurevich, 2000).

Una asimilación enriquecedora del sistema teórico marxista en su anclaje con el campo específico de los estudios históricos en comuni- 
cación, sería perceptible en la obra del destacado historiador marxista británico Raymond Williams. La validez de sus proyecciones, para el análisis histórico de las prácticas y procesos comunicativos, ubican a este autor como exponente de una de las más fructíferas vertientes dentro de la historiografía marxista de la comunicación. El denominado "materialismo cultural", enfoque teórico centrado en el análisis de las especificidades de la cultura material a partir de los presupuestos fundamentales del materialismo histórico, constituiría una corriente alternativa ante las formas tradicionales de abordar el estudio histórico de las prácticas y procesos comunicativos. Ello partiría de un desmontaje de ciertos conceptos básicos y presunciones implícitas que habían resultado predominantes en este campo de estudios. Se abogaría por una reinserción, una reevaluación de la reflexión teórica en el análisis historiográfico.

Esta perspectiva teórica -cuyo mayor impacto se verificaría en el ámbito anglosajón- proporcionaría una ruptura significativa a los modos tradicionales de concebir la historia de la comunicación, reinsertando la comprensión de las prácticas culturales y comunicativas en la complejidad de los procesos sociohistóricos. Tal perspectiva propone un desplazamiento de una concepción historiográfica enfocada en la noción estrecha de medios de comunicación para abrir el espectro de las interrogantes hacia la comprensión de dichas prácticas como procesos constitutivos de la vida sociomaterial, así como a sus determinaciones y tipicidades en contextos históricos específicos. Del mismo modo, en su reconsideración de las dinámicas relacionales entre estructuras y acción social, en su reevaluación del sujeto en el proceso históricocultural, propone un rescate del estudio de los procesos de recepción y apropiación de los mensajes y formas simbólicas, así como del uso y apropiación social de las tecnologías comunicacionales como instancia analítica decisiva en el estudio histórico de las transformaciones y (dis)continuidades en el ámbito comunicativo.

Se observa, entonces, una creciente complejización analítica, manifiesta en el campo de los estudios históricos en comunicación. Ella correspondería, por una parte, a los trayectos de su progresiva articulación en cuanto campo de estudios y, por otra, a la apropiación e influencias de paradigmas historiográficos distanciados de la visión 
positivista hegemónica. Esta evidencia pareciera justificar la certeza con que algunos autores insisten en reconocer (o conferir) un estatuto de madurez disciplinar a este campo de estudios (Montero, et al., 2001; Yanes, 2003); sin desconocer las reformulaciones comprobadas en este, dichas afirmaciones resultan en exceso optimistas; parecen obviar algunas de las carencias y desafíos fundamentales que todavía enfrenta tal campo.

\section{BALANCE CRÍTICO Y DESAFÍOS ESTRATÉGICOS: LAS NECESARIAS (RE)ORIENTACIONES FUTURAS}

Se ha indicado que el cometido fundamental de la historia de la comunicación, como proyecto disciplinar, radica en la "identificación y clarificación de los distintos fenómenos que aglutina el concepto de comunicación social, y de qué modo se incardinan en el contexto social que los genera". Su función fundamental debe ser la de contribuir a "fijar y demarcar el objeto de estudios de las ciencias de la comunicación (Ruiz, 1998, p. 397). De esta manera, le ha sido conferida una responsabilidad epistemológica esencial para el campo científico de la comunicación social.

Para la propia fundamentación científica de este campo de estudio:

La cuestión fundamental reside en la desnaturalización de los procesos comunicacionales, es decir, en la comprensión de que la significación de un fenómeno social no puede ser alcanzada si no es mediante la delimitación de su singularidad histórica. Esto equivale a extraer todas las consecuencias de la afirmación de que la comunicación no es, y no debe ser tratada como, un proceso transhistórico (Martino, 2001, p. 86).

Corresponde, entonces, abogar por un reposicionamiento de la historicidad como principio epistemológico básico en el campo científico de la comunicación. Es en este sentido que podrían ser concebidas las contribuciones básicas y las orientaciones cardinales dentro del campo de los estudios históricos en comunicación. Sin embargo, una serie de limitaciones, todavía esenciales, constriñen notablemente las potenciales contribuciones de esta área de estudios. 
De una parte, la estructuración de variantes autónomas en función de la diversidad de medios o actividades profesionales, aunque válida, ha contribuido forzosamente a la disgregación del objeto de estudio de la historia de la comunicación social como (sub)campo disciplinar. No se trata de negar la importancia o el interés de los estudios factuales, ni de la pertinencia del estudio histórico particular de los distintos medios, instituciones, figuras y prácticas profesionales. Ellas constituyen un primer estadio necesario en el desarrollo de este (sub)campo de estudios. Sin embargo, más que en la construcción teórica de un objeto de estudio que fundamente y revele la pertinencia de su articulación como disciplina autónoma en el campo científico de la comunicación, la historia de la comunicación como (sub)campo de estudios ha colocado el énfasis en su justificación disciplinar a partir de la suma asintótica de "objetos particulares" de investigaciones y vertientes específicas. Esta propensión resulta agravada por el hecho de que dichos "objetos" han resultado, en su mayoría, preconcepciones insuficientemente construidas o develadas desde el punto de vista teórico.

A nuestro juicio, otra de las paradojas fundamentales evidenciadas en este (sub)campo de estudios consiste en el inadecuado manejo de la interdisciplinariedad. Este ámbito de estudios, en el que la interdisciplinariedad deviene un principio básico, no ha logrado consolidar un acoplamiento satisfactorio de las orientaciones conceptuales y metodológicas de sus campos matriciales. Esta puede ser considerada una aseveración osada. Sin embargo, sólo desde este reconocimiento fundamental resultan explicables algunos de sus desfases con respecto al campo de la historiografía, así como algunas de sus limitaciones con respecto al propio campo de estudios de la comunicación. La adhesión -escasamente cuestionada o problematizada- a enfoques teórico-metodológicos, criterios de demarcación temporal, principios de intelección, o categorías de análisis exportadas por analogía desde el campo de la historiografía, han inhibido y retardado la necesaria generación de constructos teóricos y categoriales específicos dentro del campo de estudios históricos en comunicación.

En la búsqueda de un estatuto de legitimidad, el campo de estudios históricos en comunicación ha privilegiado una concepción de la interdisciplinariedad asociada más a relaciones de dependencia que de 
reinvención o reapropiación creativa. De estas confusiones básicas, de este inadecuado manejo del principio de interdisciplinariedad, se ha derivado, además, el desconocimiento o desfase de este campo de estudios con respecto a algunas de las propuestas más enriquecedoras y revolucionarias de la historiografía. En síntesis, podría considerarse entonces que la interdisciplinariedad ha sido -y en parte continúa siendo- una noción engañosa, una suerte de blanco movedizo, a cuyo amparo se ha acogido tanto la adopción simplista de enfoques y perspectivas historiográficas como la inexistencia de un esfuerzo consciente y sistemático en la construcción de modelos teóricos y categorías de análisis capaces de atender a las singularidades y complejidades del estudio diacrónico de los procesos, prácticas, instituciones y sistemas de comunicación.

Por otra parte, la adopción de principios de inteligibilidad impuestos a partir de nociones configuradas y entronizadas en el presente, ha implicado la renuncia a una auténtica comprensión de las tipicidades y singularidades de su configuración histórica. La tendencia ha sido naturalizar los procesos comunicacionales modernos como si se tratasen de elementos o instancias independientes del sistema de relaciones sociales que intervienen en su configuración. Algunas de las orientaciones centrales que han guiado la estructuración de este (sub)campo de estudios han descansado en usos ambiguos o en definiciones no explicitadas o construidas en torno al concepto central de comunicación, que remiten a algunas presunciones epistemológicas subyacentes en el campo científico de la comunicación. En este sentido, Martino (2001, p. 88) indica cómo interpretaciones pseudohistóricas de su propio objeto de estudio muestran un problema de "falsa homonimia" que "presupone la comunicación como entidad, siempre igual e idéntica a sí misma a lo largo del tiempo".

La búsqueda de una auténtica madurez científica en el campo de los estudios históricos en comunicación deberá considerarse a partir de una ruptura legítima con algunos de los principales patrones evidenciados en su tradición investigativa. Su mayor autonomía y maduración como (sub)campo de estudios dependerá de su capacidad para la generación o adopción fecunda de categorías de análisis, cuya potencialidad explicativa y heurística contribuya a hacer inteligible su objeto de estudio, en articulación con el entramado complejo de relaciones e instancias que intervienen en su configuración histórica. 
De este modo, lo que está en juego no son, únicamente, las demandas efectivas de un (sub)campo de estudio que debe proponerse dar cuenta de "la transformación misma del sentido de los procesos comunicacionales" (ibidem, p. 89), sino también -y sobre todo- el modo en que estas pueden contribuir a la comprensión y análisis del devenir histórico-social en general.

\section{Bibliografía}

Aróstegui, J. (2001). La investigación histórica: teoría y método. Barcelona: Editorial Crítica.

Atwood, R. (1978). New Directions for Journalism Historiography. Journal of Communications Inquiry, 12: 49. Recuperado el 10 de mayo de 2007 de la base de datos SAGE.

Barbosa, M. (2001). Medios de comunicación y conmemoraciones. Estrategias de reactualización y construcción de la memoria. Revista Signo y Pensamiento, 39, 104-112.

Barbosa, M. (2006). Meios de Comunicação no Brasil Pós-30: reflexões em torno da historicidade e do papel da imprensa. Recuperado el 19 de septiembre de 2007 de la página web de ALAIC.

Brennen, B. (1993). Newsworker in fiction: Raymond Williams and Alternative Communication History. Journal of Communication Inquiry, 17: 95. Recuperado el 21 de noviembre de 2007 de la base de datos SAGE.

Capparelli, S. \& Stumpf, I. R. C. (2001). El campo académico de la comunicación, revisitado. En R. Fuentes \& M. I. Vassallo (Comps.), Comunicación: campo y objeto de estudio.Perspectivas reflexivas latinoamericanas (pp. 59-73). Guadalajara: Universidad de Guadalajara.

Del Palacio, C. (2000). Historiar los medios de comunicación en México, un grito desde la marginalidad. Revista Universidad de Guadalajara.

Fuentes, R. \& Sánchez, E. (1989). Algunas condiciones para la investigación científica de la comunicación en México. En Cuadernos Huella, 17. Guadalajara: ITESO.

Fuentes, R. \& Sánchez, E. (1992). El estudio de la comunicación desde una perspectiva sociocultural en América Latina. Revista Diálogos de la Comunicación, 32. 
Fuentes, R. (1999). La investigación de la comunicación en América Latina: condiciones y perspectivas para el siglo XXI. Revista Diálogos de la Comunicación, 56, 52- 68.

Gargurevich, J. (2000). ¿Qué historia de la información y cómo enseñarla? Recuperado el 21 de septiembre del sitio de ALAIC.

Gil, G. J. (2001). Epistemología y estudios de comunicación. En R. Fuentes \& M. I. Vassallo (Comps.), Comunicación: campo y objeto de estudio. Perspectivas reflexivas latinoamericanas (pp. 91-104). Guadalajara: Universidad de Guadalajara.

Herrera, B. (2001). Historia de la comunicación como oficio. Apuntes sobre teoría y método. Revista Signo y Pensamiento, 39, 7-14.

Kayser, J. (1961). El Periódico: estudios de morfología, de metodología y de prensa comparada. Quito: Ediciones CIESPAL.

Martino, L. C. (2001). Elementos para una epistemología de la comunicación. En R. Fuentes \& M. I. Vassallo (Coords.), Comunicación: campo y objeto de estudio. Perspectivas reflexivas latinoamericanas (pp. 7590). Guadalajara: Universidad de Guadalajara.

Montero, D., J. \& Rueda, J. C. (2001). Introducción a la historia de la comunicación social. Barcelona: Ariel Comunicación.

Nerone, J. (s/a). Approaches to Media History. Institute of Communications Research. Illinois: University of Illinois. (versión digital).

Pizarroso, A. (1998). La historia de la propaganda: una aproximación metodológica. Revista Historia y Comunicación Social, 4, 145171.

Román, P. M. (2000). Aspectos metodológicos de la historia de la comunicación. Revista Ámbitos, 5, 119-128.

Ruiz, M. J. (1998). Notas para el estudio del origen de la comunicación social. Revista Historia y Comunicación Social, 3, 391-401.

Sánchez, E. (2002). La investigación latinoamericana de la comunicación y su entorno social: notas para una agenda. Revista Diálogos de la Comunicación, 62, 24-36.

Schudson, M. (1993). Enfoques históricos en los estudios sobre comunicación. En K. B. Jensen \& N. W. Jankowski (Coords.), Metodologías cualitativas de investigación en comunicaciones de masas. Barcelona: Casa Editorial Bosch Comunicación. 
Timoteo, J. (1988). Historia y modelos de la comunicación en el siglo $X X$. La Habana: Editorial Pablo de la Torriente.

Vassallo, M. I. (1999). La investigación de la comunicación: cuestiones epistemológicas, teóricas y metodológicas. Revista Diálogos de la Comunicación, 56, 12- 27.

Williams, R. (1980). Marxismo y literatura. Barcelona: Ediciones Península.

Williams, R. (Coord.), (1992). Historia de la comunicación. Barcelona: Casa Editorial Bosch Comunicación.

Yanes, J. A. (2003). La renovación de la historiografía de la comunicación social en España. Revista Historia y Comunicación Social, 8, 241-258. 
